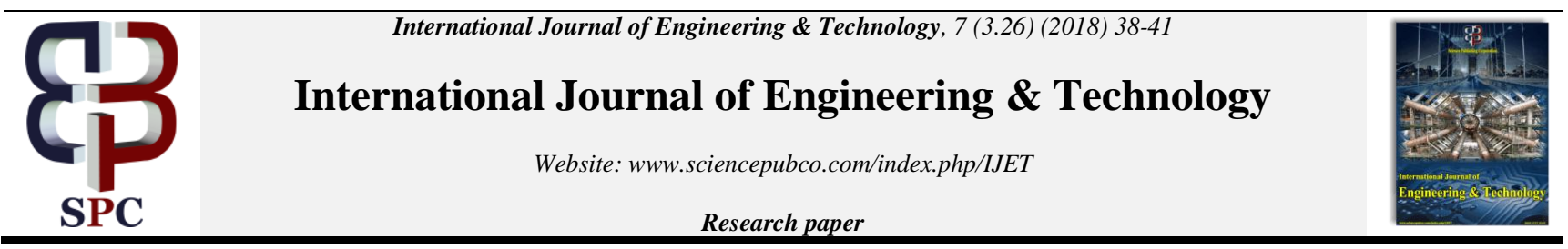

\title{
Studies on Closed-loop Interaction in a Multi-loop Single Tank Control System
}

\author{
Abhijeet Chourdhary ${ }^{1}$, Amit Jain ${ }^{2} *$ \\ ${ }^{1,2}$ Department of Chemical Engineering, BITS Pilani, Pilani - Campus, Pilani - 333031 (Rajasthan), \\ *Corresponding author E-mail:amitjain@ pilani.bits-pilani.ac.in
}

\begin{abstract}
This paper investigates the interaction between level and flow loop in a single tank system. The data generated is used to generate all transfer functions via step test method. The model generated is then simulated on MATLAB-Simulink and the obtained results are then compared with experimental results for verification. A Relative Gain Array analysis is performed to check the interaction and comment on the pairing.
\end{abstract}

Keywords: MIMO Process; Relative Gain Array; Step Test Method;Simulink

\section{Introduction}

A MIMO is a process in which there are multiple inputs and multiple outputs and each one of them can influence the other. Most of the industrial processes in chemical industry revolve around multi-input, multi-output process which cannot be understood with the principles established for single-input, single output process. The problem with process control of MIMO processes is the interaction between loops and how to regulate them. A MIMO process due to interactions between different inputs and outputs raises the question of pairing of each of these processes variable to each other so that we get a process with least interaction and highest stability (Liu, Zhang, and Gu, 2005). To control a MIMO process a generalized approach in the industry is to use $n$ PI or PID controllers for $\mathrm{n}$ control variables (Gagnepain and Seborg, 1982). Given this approach is so widely used in industry, we will also design our experiment according to this heuristic.

To study a multi-loop control system, we designed a single tank system with level and flow loop on a Universal Process Trainer. A Universal process control trainer helps understand the process control of various industrial systems by providing us with different tools which include control valves, controllers for different variables, variable transmitters and many other instruments which help us to study different systems generally seen in an industrial unit (Apex 2012).

\section{Analysis and Design}

\section{A. Block Diagram Analysis}

Block diagram analysis compacts all the gain functions of a process in a matrix. For a MIMO process, this tool helps in the analysis of gain function of respective loops and disturbance from interactions between loops.

A $2 \times 2$ MIMO process will have four gain functions as represented in the figure below. Figure 1 shows a process which is on 1-1/2-2 controller setting. Our system will be configuring for 1-1/2-2 controller setting. There is an alternate pairing scheme too known as $1-2 / 2-1$ controller setting. In this setting input 1 is controlling output 2 and input controlling output 1 as shown in Figure 2.

\section{B. Ziegler Nicholas Tuning Method}

PID controllers are the most widely used controller in industry. A common heuristic approach in tuning these controllers is Ziegler Nicholas method (Acosta, Mayosky, and Catalfo, 1994). This heuristic is only applied when the system is in closed configuration.

A brief summary of the process as given in the original paper (Ziegler and Nichols, 1993):

1. Turn off the derivative and integral action of the controller.

2. Increase the proportional control from zero till the process reaches a quasi-static state.

3. Note down the critical gain and critical time period.

4. Use the Z-N table to tune your controller.

As an accepted heuristic, this method was used in the experimental setup to tune the PIDs of both controllers. Although Z-N heuristic is not the most reliable method for tuning of PID controller (Acosta et al., 1994) it did give acceptable results and further tweaking improved the action of both the controllers.

\section{Relative Gain Array Method}

Pairing and interaction are the two most important questions in a MIMO process. Bristol in 1966 developed a systemic approach 


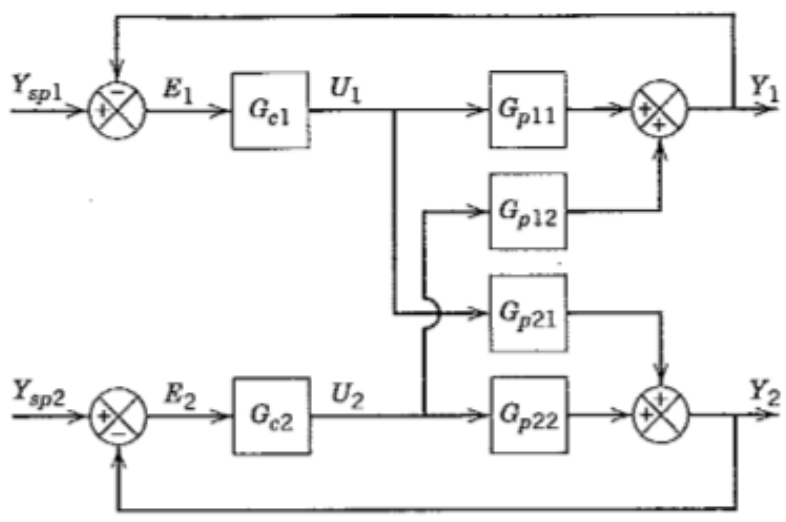

(a)

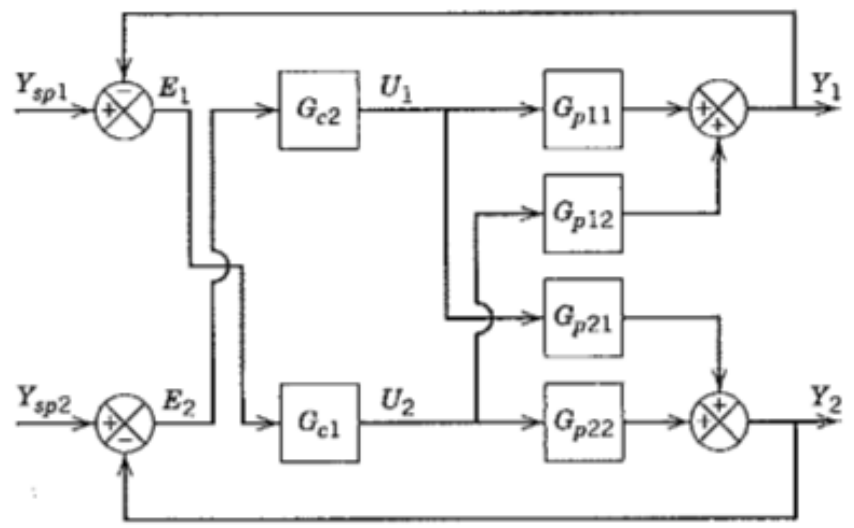

(b)

Fig. 1: A block analysis of a 2×2 MIMO process in (a)1-1/2-2 pairing; (b) 1-2/2-1 pairing (Seborg et al., 2004).

called Relative Gain Array Method to answer these questions (Mc Avoy et al., 2001). This method relied on the concept of relative gain. Bristol considered a process with $\mathrm{n}$ controlled variables and $\mathrm{n}$ manipulated variables, relative gain $\lambda_{\mathrm{ij}}$ was defined as gain between a controlled variable, $Y_{i}$ and manipulated variable, $U_{j}$ which equals steady state gain of process in two states (Seborg et al., 2004).

$\lambda_{i j}=\frac{\left(\frac{\partial y_{i}}{\partial u_{j}}\right)_{u}}{\left(\frac{\partial y_{i}}{\partial u_{j}}\right)_{u}}=\frac{\text { open-loopgain }}{\text { closed-loopgain }}$

Assume a $2 \times 2$ process with $u$ as input and $y$ as output. RGA of this process will be:

$$
\Lambda=\left[\begin{array}{cc}
\lambda & 1-\lambda \\
1-\lambda & \lambda
\end{array}\right]
$$

where,

$$
\lambda=\frac{1}{1-\frac{k_{12} k_{21}}{k_{11} k_{22}}}
$$

where, $\kappa$ is steady state gain.

According to the calculated value of $\lambda$ comments are made about the interaction between loops as described in Table 1 .

Table 1. Relative Gain Array Matrix of a 2 x 2 process

\begin{tabular}{|l|l|}
\hline \multicolumn{1}{l|}{ (Seborg et al., 2004) } \\
\hline$\lambda \geq 0.5$ & Recommended Configuration \\
\hline$\lambda<0.5$ & $1-1 / 2-2$ configuration \\
\hline
\end{tabular}

\section{Step Test Method}

Ziegler and Nicholas invented two tuning methods, frequency test and step test (Astrom and Hugglund, 2004). The method stated above is frequency analysis. Step test is done on an open loop. A brief summary of step test method (Ziegler and Nichols, 1993):

1. Let the process stabilize to a steady state.

2. Turn off the PID controllers.

3. Give a step input to the process.

4. Wait for the process to stabilize.

5. If the process is unstable in open loop, step test cannot be applied.

6. Calculate the model parameters using the graphical method.

By using step test method, transfer functions are generated for the experimental setup.

\section{E. Simulation}

The process is then simulated on MATLAB Simulink and compared with the experimental values collected. The simulation used standard process protocols provided by MATLAB and MATLAB based tools for graphical analysis.

The simulation has two step input blocks so as to control the input of both the loops independently. Both step inputs are followed by respective PID controllers with feedback as the output of each loop. The output of controllers is then modified using transfer function already identified with their respective delays. The output generated is then given to scope for immediate graphical analysis and to *.mat file generator for exporting the output.

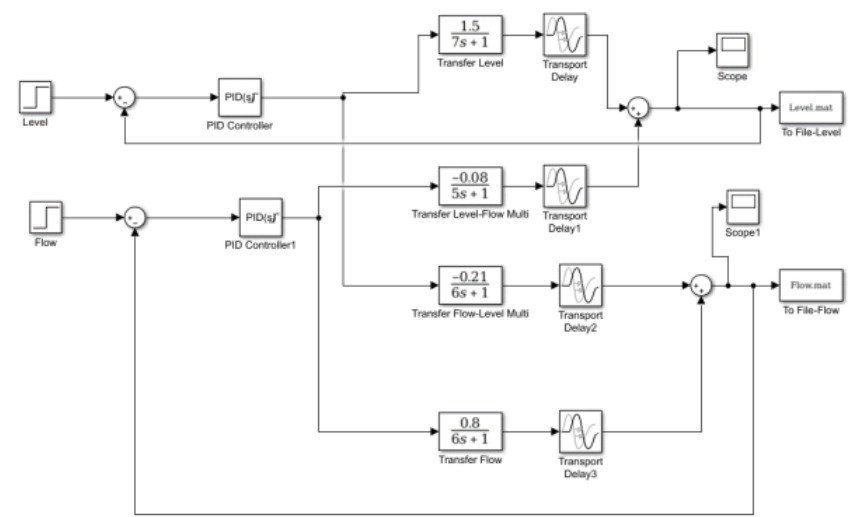

Fig. 2: Flowsheet of the Simulated Model

\section{Experiments, Results and Discussion}

\section{A. Stability of loop response after Z-N Tuning}

Both the controllers were tuned using Z-N tuning analysis. Although response curves were still not stable after tuning, the values obtained from Z-N tuning were modified till stable response curves were required for both loops as shown in Fig. 3 and 4.

Responses of both the loops were recorded for more than 2 hours at a step input of 0.5 or $50 \%$.

\section{B. Interaction Experiments}

After tuning of controllers, interaction experiments were performed to confirm interaction between loops as shown in Fig. 5 and 6.

\section{Step Test and Transfer Functions}

Step Test was performed on both the loops in open loop configuration. Graphical analysis is shown in Fig. 3 and Fig. 4. 


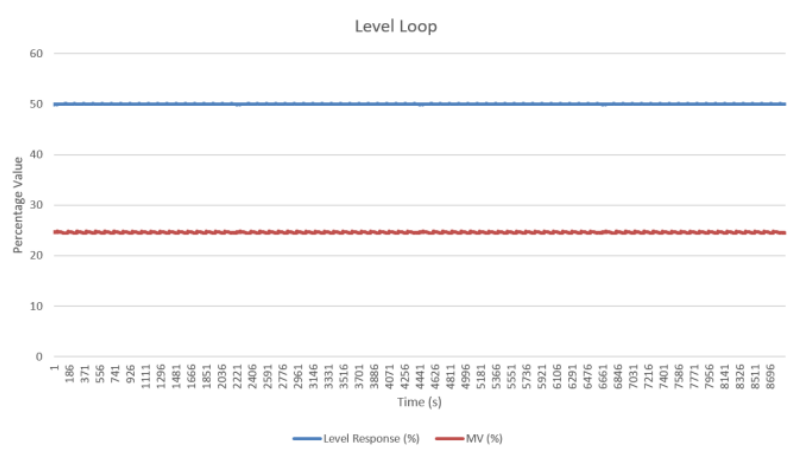

Fig. 3: Response Curve of Level Loop

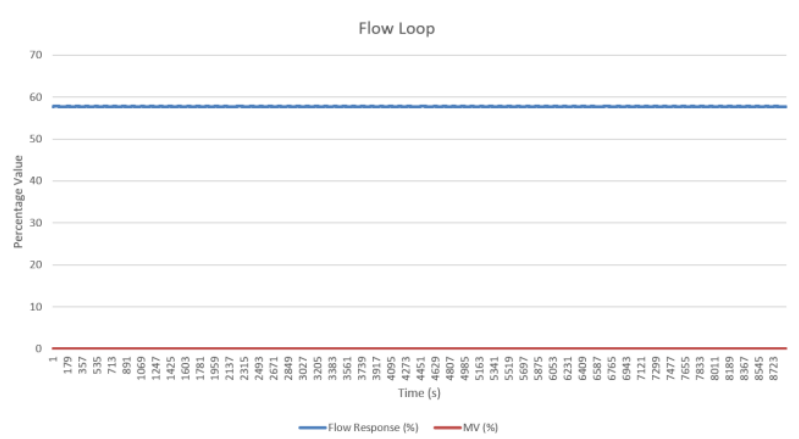

Fig. 4: Response Curve of Flow Loop

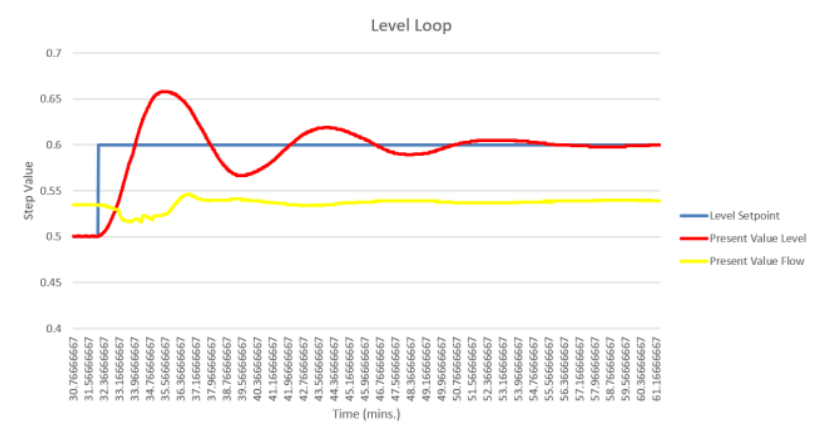

Fig. 5: Response of Level and Flow loop to step change in Level Loop

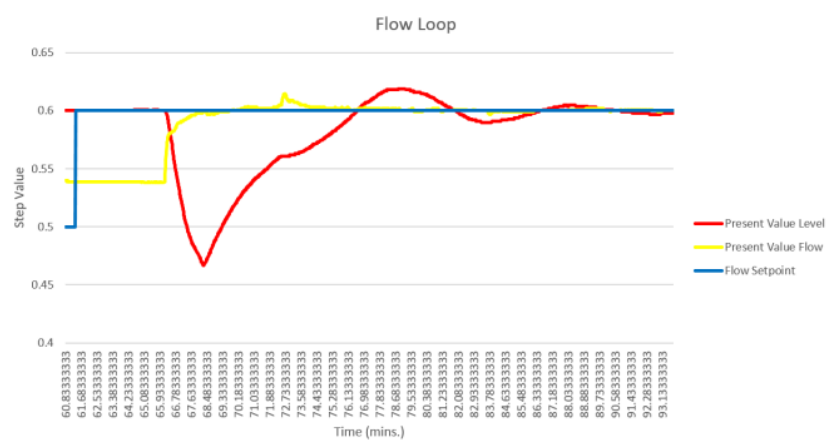

Fig. 6: Response of Level and Flow loop to step change in Flow Loop

\section{Transfer Functions}
$G_{p 11}=\frac{1.5 e^{-1.5 s}}{7 s+1}$
$G_{p 12}=\frac{-0.08 e^{-0.75 s}}{5 s+1}$
$G_{p 21}=\frac{-0.21 e^{-3 s}}{6 s+1}$
$G_{p 22}=\frac{0.8 e^{-0.08 s}}{6 s+1}$

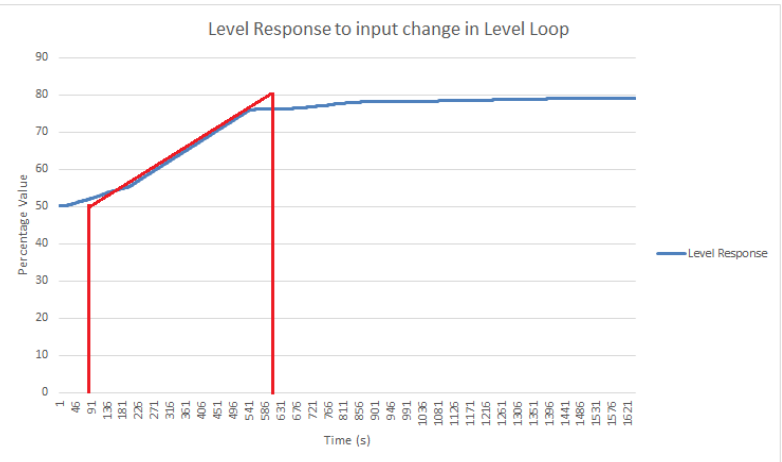

(a) Response of Level Loop to step change in Level Loop

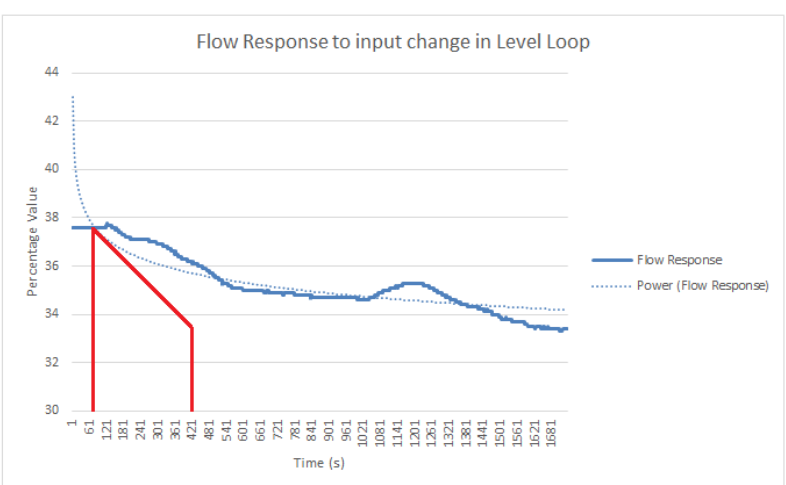

(b) Response of Flow Loop to step change in Level Loop

Fig. 7: Graphical Analysis of response of Level Loop (Open Configuration) and Flow Loop (Open Configuration) to step change in Level Loop.

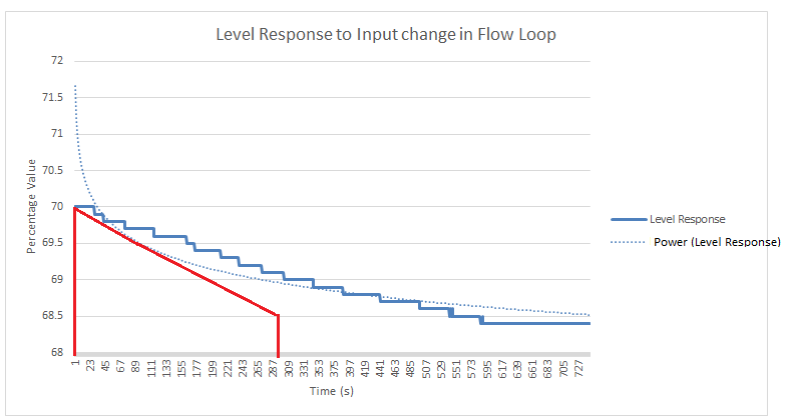

(a) Response of Level Loop to step change in Flow Loop

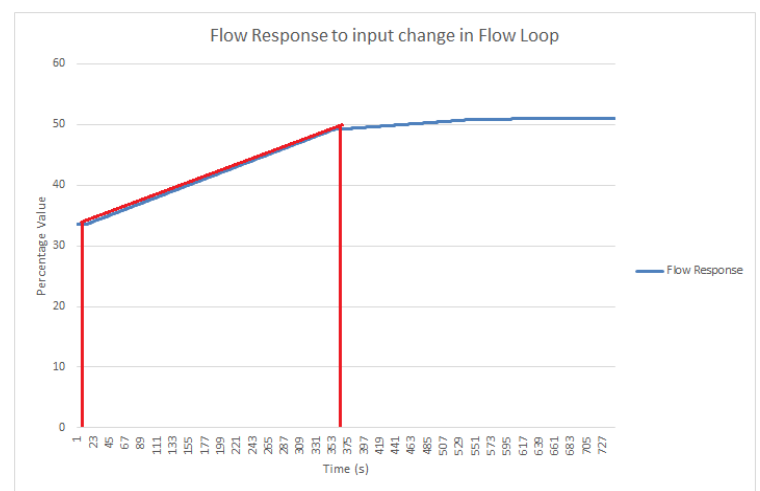

(b) Response of Flow Loop to step change in Flow Loop

Fig. 8: Graphical Analysis of response of Level Loop (Open Configuration) and Flow Loop (Open Configuration) to step change in Flow Loop

0.5. So, according to RGA heuristics, the model has the least interaction when operated in 1-1/2-2 pairing configuration.

F. Simulation Results

A similar set of interaction experiments were performed on the model generated. 


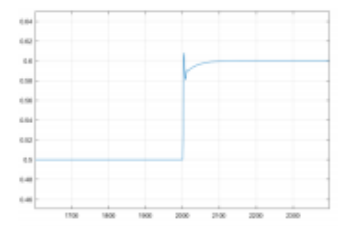

(a) Response of Level Loop to step change in Level Loop

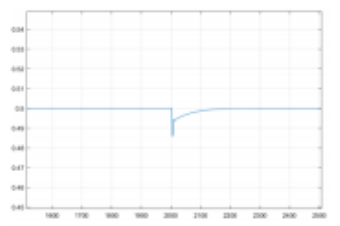

(b) Response of Flow Loop to step change in Level Loop

Fig. 9: Comparative Graphical analysis of both Responses

\section{G. Discussion}

The following points discuss the result obtained:

1. Model generated predicts correctly the nature of response for a given step change but is not precise with quantification of the response generated in the loop.

2. The model generated is a linearized approximation of the experimental setup and this might be the reason for the incorrect prediction of amplitude of response.

3. RGA analysis of the setup suggests a 1-1/2-2 configuration for the least interaction setup which is already followed.

\section{Conclusion}

We can conclude from our set of experiments and discussion that linearized model of a level-flow multi-loop system although predicts the nature of response, can be improved for quantitative predictions and 1-1/2-2 configuration gives the least interaction pairing of a level-flow multi-loop system.

\section{References}

[1] Gerardo Gabriel Acosta, Miguel Angel Mayosky, and Jose’ Maria Catalfo (1994). "An expert PID controller uses refined Ziegler and Nichols rules and fuzzy logic ideas". Applied Intelligence 4 (1), pp. 53-66. ISSN: 1573-7497. DOI: 10.1007/BF00872055. URL: https://doi.org/10.1007/BF00872055.

[2] Apex. Universal Process Control Trainer Manual. Apex Innovations Pvt. Ltd., 2012.

[3] Thomas Mc Avoy, Yaman Arkun, Rong Chen, Derek Robinson and P David Schnelle (2003). "A new approach to defining a dynamic relative gain". Control Engineering Practice 11 (8), pp. 907-914. DOI: https://doi.org/10.1016/S0967-0661(02)00207-1.

[4] Jean Pierre Gagnepain and Dale E. Seborg (1982). "Analysis of process interactions with applications to multiloop control system design”. In: Industrial \& Engineering Chemistry Process Design and Development 21 (1), pp. 5-11. DOI:10.1021/i200016a002.

[5] Tao Liu, Weidong Zhang, and Danying Gu (2005). "Analytical multi-loop PI/PID controller design for $2 \times 2$ processes with Time Delays". Industrial \& Engineering Chemistry Research 44 (6), pp. 1832-1841. DOI: 10.1021/ie0493715.

[6] Dale E Seborg, Thomas F. Edgar, and Dunkan A. Mellichamp (2008). Process Dynamics and Control. 2nd Edition. John Wiley Sons, Inc.

[7] J.G. Ziegler and N.B. Nichols (1993). "Optimum Setting for Automatic Controllers". pp. 759-768.

[8] K.J. Astrom and T. Hugglund (2004). "Revisiting the ZieglerNichols step response method for PID control". Journal of Process Control 14 (6), pp. 635-650. DOI: https://doi.org/10.1016/ j.jprocont.2004.01.002. 